\title{
Increased reactive oxygen species levels cause ER stress and cytotoxicity in andrographolide treated colon cancer cells
}

\author{
Aditi Banerjee $^{1}$, Vivekjyoti Banerjee ${ }^{1}$, Steven Czinn ${ }^{1}$, Thomas Blanchard ${ }^{1}$ \\ ${ }^{1}$ Department of Pediatrics, University of Maryland School of Medicine, Baltimore, Maryland, U.S.A \\ Correspondence to: Aditi Banerjee, email: abanerjee@peds.umaryland.edu \\ Keywords: andrographolide, chemotherapy, reactive oxygen species, endoplasmic reticulum stress, unfolded protein \\ response \\ Received: August 15, 2016 \\ Accepted: January 30, 2017 \\ Published: February 16, 2017 \\ Copyright: Banerjee et al. This is an open-access article distributed under the terms of the Creative Commons Attribution License (CC-BY), \\ which permits unrestricted use, distribution, and reproduction in any medium, provided the original author and source are credited.
}

\section{ABSTRACT}

Chemotherapy continues to play an essential role in the management of many cancers including colon cancer, the third leading cause of death due to cancer in the United States. Many naturally occurring plant compounds have been demonstrated to possess anti-cancer cell activity and have the potential to supplement existing chemotherapy strategies. The plant metabolite andrographolide induces cell death in cancer cells and apoptosis is dependent upon the induction of endoplasmic reticulum stress (ER stress) leading to the unfolded protein response (UPR). The goal of the present study was to determine the mechanism by which andrographolide induces ER stress and to further evaluate its role in promoting cell death pathways. The T84 and COLO 205 cancer cell lines were used to demonstrate that andrographolide induces increased ROS levels, corresponding anti-oxidant response molecules, and reduced mitochondrial membrane potential. No increases in ROS levels were detected in control colon fibroblast cells. Andrographolide-induced cell death, UPR signaling, and CHOP, Bax, and caspase 3 apoptosis elements were all inhibited in the presence of the ROS scavenger NAC. Additionally, andrographolide-induced suppression of cyclins B1 and D1 were also reversed in the presence of NAC. Finally, Akt phosphorylation and phospho-mTOR levels that are normally suppressed by andrographolide were also expressed at normal levels in the absence of ROS. These data demonstrate that andrographolide induces ER stress leading to apoptosis through the induction of ROS and that elevated ROS also play an important role in down-regulating cell cycle progression and cell survival pathways as well.

\section{INTRODUCTION}

The phytochemical andrographolide (Andro), is a bicyclic diterpenoid lactone purified from Andrographis paniculata, a herb widely used throughout Asia as a traditional remedy for numerous maladies. It has been used extensively to treat both infectious and chronic diseases [1], and both in vitro and in vivo experimental models provide detailed evidence that Andro possesses potent anti-inflammatory properties [2]. Andrographolide has also been demonstrated to possess multifaceted anticancer cell activity and has been tested against human cells from breast cancer [3, 4], lung cancer [5, 6], leukemia [7], colon cancer [8,9], liver cancer [10,11], prostate cancer $[12,13]$, and others. These models have been used to determine that Andro activates pro-apoptosis pathways and induces cell cycle arrest at both the G1/S and G2/M phases. Studies in vivo employing murine xenograft models of human cancers have yielded positive results when treated with Andro demonstrating delayed tumor growth when applied either alone or in combination with other chemicals [14-17]. Although many studies describe the various signaling events leading to apoptosis and measure the factors that regulate cell cycle progression in the context of Andro treatment, little is known about the early cellular events following Andro treatment that lead to these events.

We recently reported that Andro-induced cell death occurs via ER stress in colon cancer cells as demonstrated by blocking the unfolded protein response (UPR) [18]. While ER stress can initiate downstream signaling leading to apoptosis via the IRE-1, PERK, and ATF6 ER membrane proteins, we observed that Andro-induced cell cytotoxicity occurred primarily through IRE-1 activity as shown by over expression of IRE-1 as well as depletion of IRE-1 with siRNA. The ER stress response is best 
understood in the context of an accumulation of unfolded or incorrectly folded proteins [19]. The cell responds to such alterations through the UPR in which proteins such as GRP78, IRE-1, PERK, and ATF6 transmit signals to activate mechanisms to ameliorate the accumulation of the altered proteins. When ER stress becomes irreversible, these same pathways will promote apoptosis to eliminate the cells.

Many factors can contribute to the induction of ER stress and the UPR including over-expression of proteins beyond the capacity of the ER to correctly fold them, inhibition of glycosyation [20], ER $\mathrm{Ca}^{2+}$ depletion, and oxidative stress among others. We now report that Andro induced ER stress/UPR leading to apoptosis is dependent upon the induction of oxidative stress. Andro induces reactive oxygen species (ROS) along with expression of multiple antioxidant response genes. Inhibition of ROS significantly reduces expression of UPR proteins as well as cell death and proapoptosis pathways. We also report that in addition to inducing apoptosis via the UPR, Andro blocks Akt phosphorylation resulting in decreased levels of mTOR, and suppresses Cyclins B1 and D1 of the cell cycle progression pathway. Scavenging of Androinduced ROS blocked these activities. These data provide additional insight into the anticancer cell activity of Andro.

\section{RESULTS}

\section{Andrographolide selectively inhibits colon cancer cells}

The MTT assay was used to evaluate the effects of Andro on colon cancer COLO 205 cell numbers when treated for up to $72 \mathrm{~h}$. There was a dose and time dependent inhibition of cell viability (Figure 1A) The $\mathrm{IC}_{50}$ at 24,48 and $72 \mathrm{~h}$ was determined to be 80,45 , and $26 \mu \mathrm{M}$ respectively. Treatment of normal colon epithelial cells with the same concentration of Andro had little effect on cell numbers which only dropped below $80 \%$ at the highest dose tested (Supplementary Figure 1). These data suggest that Andro selectively inhibits colon cancer cells but not normal colon cells. These results were consistent with our previous report utilizing T84 and HCT 116 colon cancer cell lines to test Andro activity and the $\mathrm{IC}_{50}$ $(45 \mu \mathrm{M})$ at $48 \mathrm{~h}$ was used for subsequent assays. FDAPI double staining of Andro treated COLO 205 cells revealed the incorporation of less FDA and increased PI staining indicating increased cell death relative to untreated control cells (Figure 1B). To determine whether the Andro associated decreased viability was due to the induction of apoptosis, nuclear morphology was examined by microscopy using DAPI staining. Treatment of COLO 205 cells with Andro $(45 \mu \mathrm{M})$ for $24 \mathrm{~h}$ and $48 \mathrm{~h}$ revealed several apoptotic morphological features including apoptotic bodies, cell shrinkage, and chromatin condensation not observed in control cells, most evident at $48 \mathrm{~h}$
(Figure 1C). The degree of apoptosis was assessed further by quantitative ELISA to measure apoptotic nucleosomes. Nucleosomes present in cell lysates following $48 \mathrm{~h}$ treatment with Andro increased in a dose dependent manner (Figure 1D). Additionally, caspase-3 activity was also found to be increased significantly in Andro-treated COLO 205 cells at $48 \mathrm{~h}(P<0.001)$ (Figure 1E).

\section{Andrographolide treatment of colon cancer cells is associated with ROS generation and loss of mitochondrial membrane potential}

Andrographolide induced cell death in Colon T84 and HCT 116 cancer cell lines has been shown to be dependent upon ER stress, specifically through IRE-1 signaling leading to CHOP expression and the induction of apoptosis [18]. The same events were observed in Andro-treated COLO 205 cells as GRP78, IRE-1, and CHOP expression were significantly increased at both 24 and $48 \mathrm{~h}$ and this activity could be inhibited with the ER stress inhibitor 4-PBA (Supplementary Figure 2). Many factors can induce ER stress including oxidative stress, and the anti-cancer effects of certain plant metabolites have been shown to trigger ROS generation [21]. We therefore investigated the role of ROS in Andro anticancer activity. Colon cancer cell lines were treated with Andro for 24 and $48 \mathrm{~h}$ and the amount of intracellular ROS was assessed using a dichlorofluorescein (DCF) based assay. Andrographolide increased ROS generation in both T84 and COLO 205 cells compared with untreated control cells by $24 \mathrm{~h}(P<0.001$ and $P<0.01$ respectively), whereas no increase was detected in human fibroblast $18 \mathrm{Co}$ cells (Figure 2A). Similar results were observed at $48 \mathrm{~h}$ (data not shown). Pretreatment of cells with the ROS inhibitor $\mathrm{N}$-acetyl-L-cysteine (NAC) $1 \mathrm{~h}$ prior to Andro treatment resulted in a significant decrease in $\operatorname{ROS}(p<0.05)$ in both colon cancer cell lines compared to cells treated with Andro alone. We next examined the role of intracellular ROS levels in the growth inhibition of colon cancer cells during Andro treatment. The ROS scavenger inhibitor NAC $(20 \mathrm{mM})$ was added $1 \mathrm{~h}$ before Andro administration and the cells were evaluated for viability by the MTT assay. The NAC pretreatment significantly inhibited Andro-induced growth inhibition in T84 and COLO 205 cells at both 24 and $48 \mathrm{~h}(P<0.001)$ (Figure 2B). Similar results were observed in a clonogenic assay where NAC pretreatment significantly increased cell viability in Andro treated cells (Figure 2C).

Generation of ROS is associated with disruption of mitochondrial membrane potential (MMP) [22]. Therefore, we investigated the effect of Andro on the integrity of the mitochondria by staining the cells with a cell permeant dye that readily accumulates in active mitochondria due to their relative negative charge. As shown in Figure 2D, incubation of T84 and COLO 205 cells with Andro caused a significant decrease of MMP at $48 \mathrm{~h}(P<0.01)$. 


\section{Andrographolide induces expression of antioxidant genes}

The up regulation of antioxidant genes can protect cells from injury due to oxidative stress. We measured transcript expression of six antioxidant genes as additional evidence that Andro induces significant ROS production. RNA was purified from T84 and COLO 205 cells at 4,6 , and $8 \mathrm{~h}$ after the addition of Andro and evaluated by real time PCR. Protein expression was measured by immunoblot or immunofluorescence. The gene expression of LPO, Nrf2, TRX, GPX, and PrX-6 in T84 cells were all significantly increased by $6 \mathrm{~h}$ (Figure $3 \mathrm{~A}$ ). However, all gene expression returned to base level by
$8 \mathrm{~h}$. Interestingly Andro had no impact on the expression of PrX-1. The response in COLO 205 cells was observed to be much narrower with only TRX and GPX increasing due to treatment with Andro (Figure 3B; $P<0.001$ ). In the case of GPX, a significant increase was seen by $4 \mathrm{~h}$ and remained high even at $8 \mathrm{~h}$. COLO 205 cells were also evaluated for antioxidant gene expression at $48 \mathrm{~h}$ of Andro treatment in the presence or absence of NAC (data not shown). The gene expression of LPO, Trx and GPx was increased greater than 1.5 fold compared to untreated cells and this activity was significantly reduced in the presence of NAC (data not shown). Trx protein was significantly elevated in Andro treated T84 at $4 \mathrm{~h}$ and in COLO 205 cells at 4 and 8 as demonstrated by
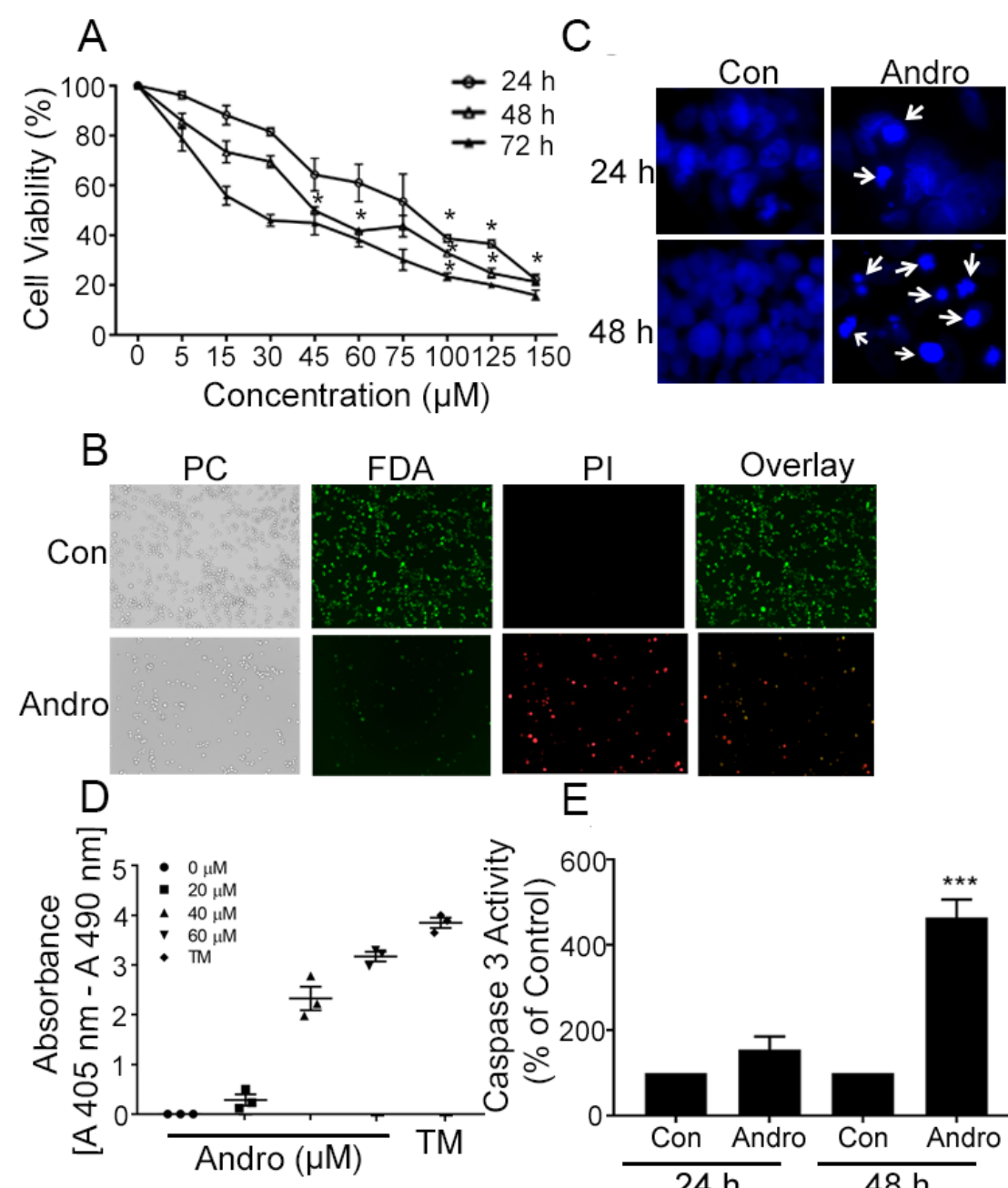

E

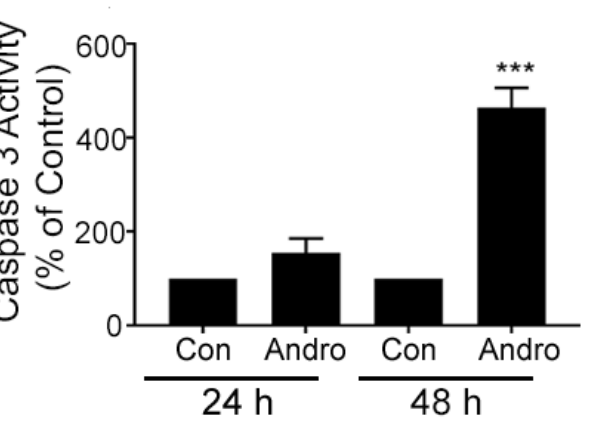

Figure 1: Andrographolide suppresses cell proliferation and cell survival in COLO 205 cells. (A) Cells were treated with Andro for 24, 48 and $72 \mathrm{~h}$ and cell viability was quantified by MTT assay. (B) Fluorescence microscopy images showing the viability of COLO 205 cells cultured in vitro with or without Andro $(45 \mu \mathrm{M})$ (left to right: phase contrast (PC) image, FDA stain, PI stain, overlay of FDA and PI stain). (C) Cells were treated with Andro $\mathrm{IC}_{50}$ dose $(45 \mu \mathrm{M})$ for either $24 \mathrm{~h}$ (upper panels) or $48 \mathrm{~h}$ (lower panels) and stained with DAPI. Apoptotic cells were identified by condensation and fragmentation (arrows) of nuclei using inverted fluorescence microscope. (D) Detection of nucleosomes in cytoplasmic fractions at increasing doses of Andro and TM. $10^{4}$ cells were treated with or without Andro $(20,40$, or $60 \mu \mathrm{M})$ or TM $(1 \mu \mathrm{g} / \mathrm{ml})$ for $48 \mathrm{~h}$ at $37^{\circ} \mathrm{C}$. Cell lysates $(20 \mu \mathrm{l})$ were analyzed in the ELISA. (E) Caspase 3 activity was evaluated by Caspase-3 colorimetric activity assay kit as described. Experiments were performed two times (D and E) or three times (A, B and C). $(* P<0.05, * * * P<0.001)$. 
immunofluorescence (Figure 3C). Immunoblot for Prx and GPx protein demonstrated transient expression in T84 cells that returned to background by $8 \mathrm{~h}$ (Figure 3D). GPx was also observed in COLO 205 cells and continued to be expressed at $8 \mathrm{~h}$.

\section{Andrographolide induced ER stress and proapoptosis signaling are ROS dependent}

Colon cancer cells were treated with Andro in the presence or absence of the ROS scavenger NAC to determine the relationship between ROS and ER stress. Immunoblot analysis for the UPR protein IRE-1 demonstrated a significant decrease in cells pretreated with NAC at both 24 and $48 \mathrm{~h}$, and for both T84 and COLO205 cells with expression returning to those observed in the absence of Andro exposure (Figure 4A). Similar results were achieved when assessing gene expression data from cells harvested at $48 \mathrm{~h}$ Andro treatment which revealed that IRE-1 levels were significantly reduced when pretreated with NAC compared to those cells receiving Andro alone for both T84 and COLO 205 cells $(P<0.001 ; P<0.01$ Figure $4 \mathrm{~B})$. The proapoptosis signaling proteins $\mathrm{CHOP}$ and XBP-1 were also reduced in the presence of NAC in both colon cancer cells compared with the NAC and
Andro treated cells (Figure 4C and 4D respectively). These data support a model in which Andro promotes ER stress leading to apoptosis signaling via the induction of ROS. These data are consistent with our previous report documenting ER stress mediated signaling by IRE-1 as quantification of gene expression for both PERK and ATF6 revealed no increase, and the anti-apoptosis molecule Bcl2 showed no increase as well (Supplementary Figure 3 ).

\section{Andrographolide induced G1/S and G2/M cell cycle arrest and apoptosis in colon cancer cells is ROS dependent}

The cell cytotoxicity activity of Andro is largely the result of apoptosis and cell cycle arrest. We therefore investigated the degree to which these two Andro induced mechanisms are dependent upon oxidative stress by evaluating the effect of Andro in the presence and absence of NAC. Immunoblot analysis was performed on Cyclin $\mathrm{A}$ and Cyclin B1, cell cycle regulatory proteins that play important roles in regulating cell cycle progression. Figure 5A shows Andro could effectively suppress Cyclin B1 expression $(P<0.001)$ at both $24 \mathrm{~h}$ and $48 \mathrm{~h}$ compared to untreated control cells in both colon cancer cell lines. Pretreatment with NAC however resulted in
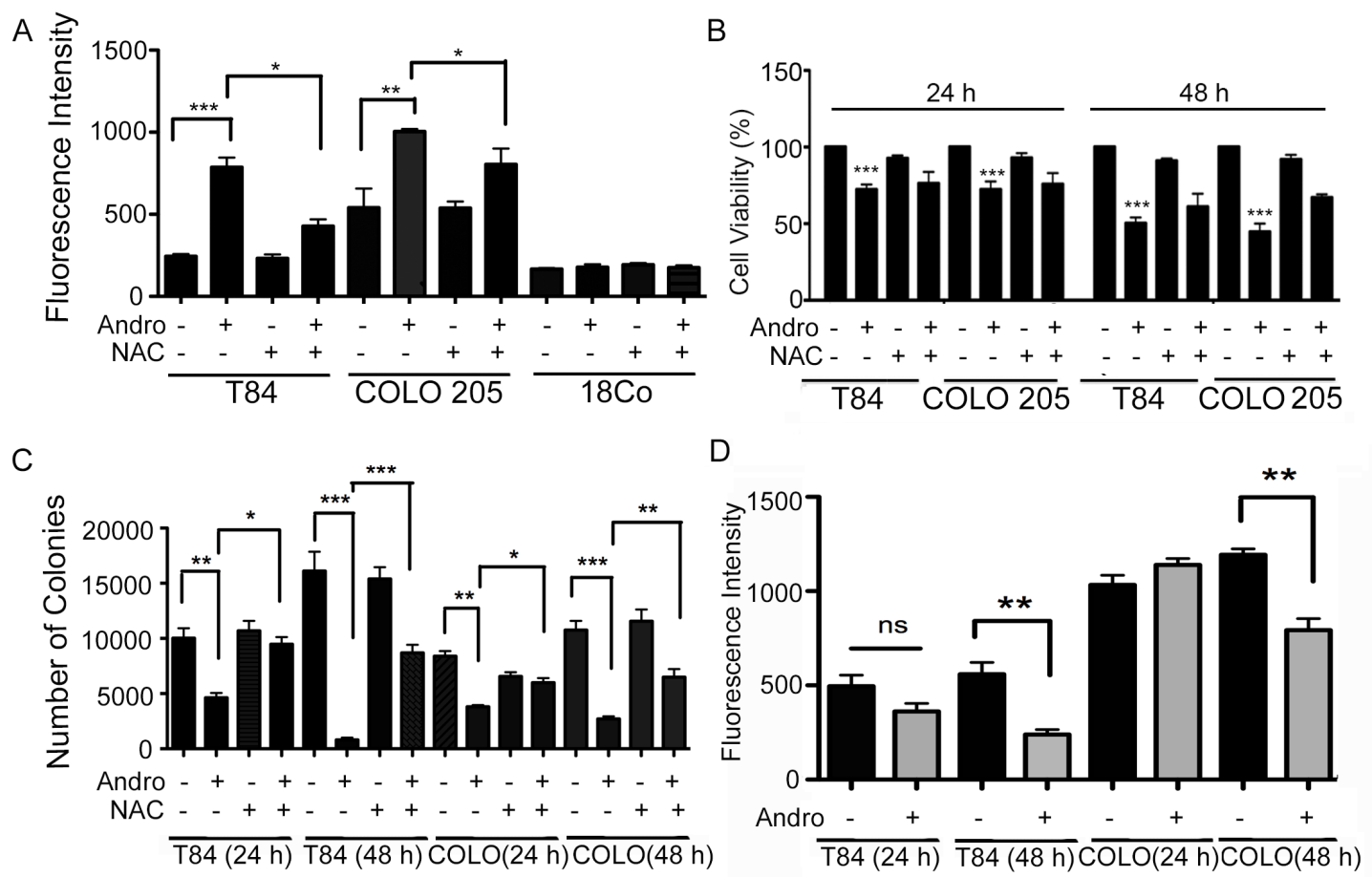

Figure 2: Andrographolide induces ROS generation in colon cancer cells. (A) The effects of Andro treatment on ROS generation. T84, COLO 205, and normal fibroblast cells $(18 \mathrm{Co})$ were treated with Andro $\mathrm{IC}_{50}(45 \mu \mathrm{M})$ for $24 \mathrm{~h}$ and cellular ROS levels were determined by measurement of fluorescent DCF. ROS inhibition was performed by pre-treatment of cells with 20 mM NAC for $1 \mathrm{~h}$ prior to Andro treatment. (B) The effects of ROS on cell viability in Andro-treated colon cancer cells were assessed by MTT assay at $24 \mathrm{~h}$ and $48 \mathrm{~h}$ in the presence of absence of NAC. Absorbance was read at $570 \mathrm{~nm}$ with averages from triplicate wells. (C) For quantification of clonogenicity Andro treated T84 and COLO 205 cells were examined by colony formation assay for $24 \mathrm{~h}$ and $48 \mathrm{~h}$ and visualized by staining with crystal violet. (D) T84 and COLO 205 cells were treated with or without Andro for $24 \mathrm{~h}$ and $48 \mathrm{~h}$ and then incubated with TMRE. Experiments were performed two times (A and D) or three times (B and $\mathrm{C})$. $(* P<0.05, * * P<0.01, * * * P<0.001)$. 


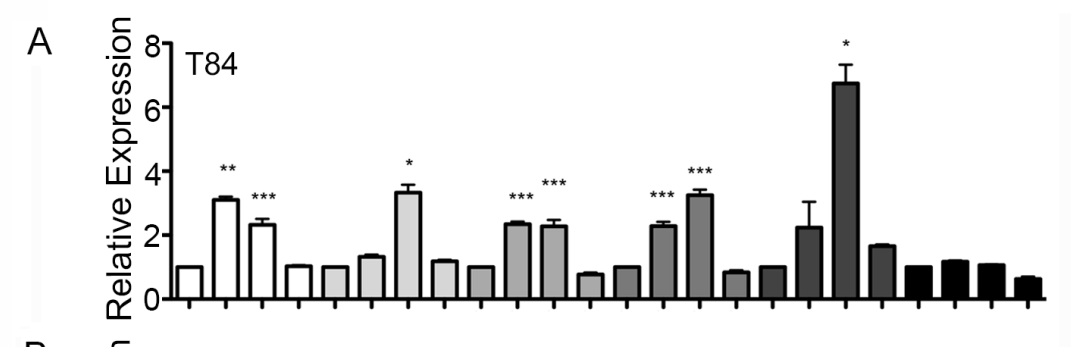

B

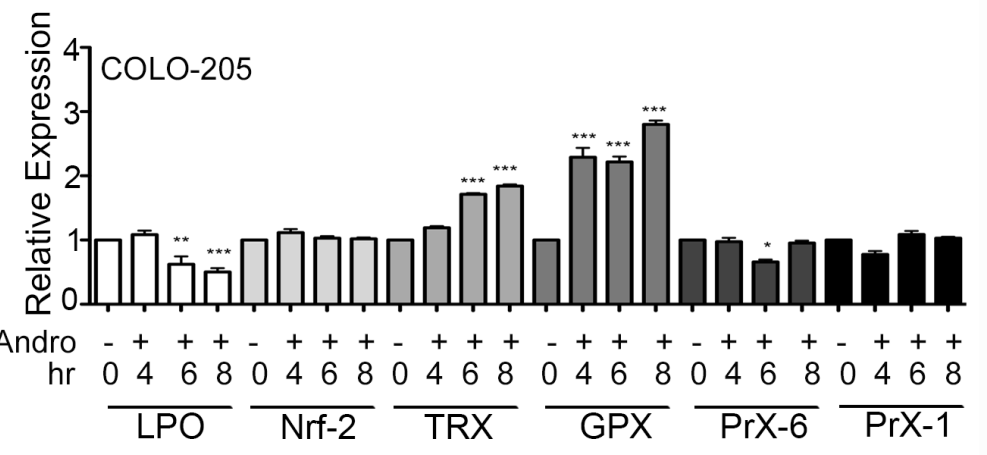

C
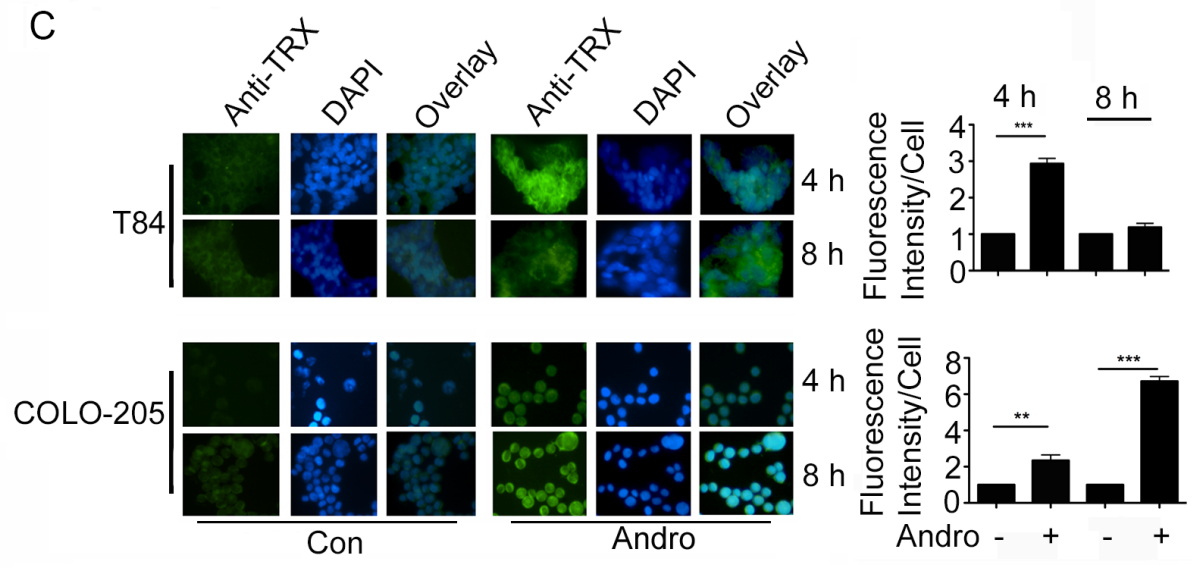

D
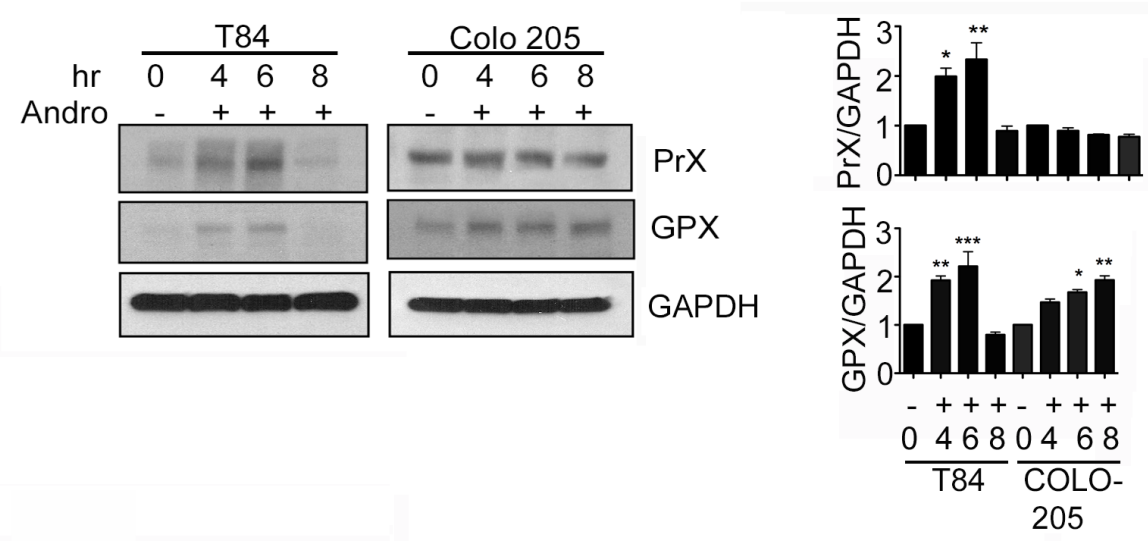

Figure 3: Andrographolide induces anti-oxidant gene expression. T84 and COLO 205 cells were treated with or without Andro at $\mathrm{IC}_{50}(45 \mu \mathrm{M})$ for 4,6 , or $8 \mathrm{~h}$ and the transcriptional level of expression for anti-oxidant genes (LPO, Nrf-2, Trx, GPx, Prx-6) were determined by qRT-PCR for (A) T84 cells and (B) COLO 205 cells. Bar graphs show quantitative results normalized to GAPDH mRNA levels. Results are from three independent experiments. (C) T84 and COLO 205 cells were grown on coverslips and treated with Andro. TRX expression was evaluated by immunofluorescent staining. Nuclei were stained using DAPI and cells were examined by fluorescence microscopy. Fluoresence intensity was determined and compared with untreated (Con) T84 and COLO 205 cells. (D) T84 and COLO 205 cells were treated with Andro for $4 \mathrm{~h}, 6 \mathrm{~h}$ and $8 \mathrm{~h}$. Cells were lysed and protein expression was determined by immunoblotting for PrX, GPX and GAPDH. Densitometry analysis was performed and normalized with GAPDH. Statistical significance was determined using one way-ANOVA followed by post hoc Tukey's test. $\left({ }^{*} P<0.05,{ }^{* *} P<0.01,{ }^{* * *} P<0.001\right)$. 
Cyclin B1 expression being significantly up regulated $(P<0.001)$ compared with cells treated with Andro alone with the exception of COLO 205 cells at $48 \mathrm{~h}$. No Andro associated increase was observed for Cyclin A. Additional analysis by real time PCR for gene expression of Cyclin D1 demonstrated a significant decrease $(P<0.001)$ in Andro treated in COLO 205 cells compared to control cells (Supplementary Figure 4). Cyclin D1 expression was observed to increase significantly and return to the levels observed in control cells when pretreated with NAC and exposed to Andro $(p<0.01)$. When caspase 3 activation was measured as an indicator for apoptosis, we observed caspase 3 activation with Andro treatment at $24 \mathrm{~h}$ and $48 \mathrm{~h}(P<0.001)$ for T84 cells while a significant activation was observed in COLO 205 cells only at $48 \mathrm{~h}(P<0.001)$. Moreover, pretreatment with NAC completely halted Andro induced caspase 3 activation in T84 cells at $24 \mathrm{~h}$ (Figure 5B). No significant induction of caspase activity was observed when normal fibroblast cells were treated with Andro (Data not shown).

\section{Andrographolide induced oxidative stress inhibits the Akt/mTOR cell survival signaling}

Andrographolide inhibits cell survival. We therefore examined the possibility that Andro also induces cell death through regulating the Akt /mTOR signaling, a major pathway in the growth and survival of cancer cells. We observed a significant Andro-induced down regulation of phospho-Akt (ser 473) and phospho-Akt (Thr 308) expression in both colon cancer cell lines at $24 \mathrm{~h}$ and $48 \mathrm{~h}$ by immunoblot (Figure $6 \mathrm{~A}$ and $6 \mathrm{~B}$ respectively). Pretreatment of the cells with NAC before Andro exposure resulted in a significant increase in phospho-Akt (ser 473) in both T84 cells and COLO 205 cells $(P<0.01$ and $P<0.05$, respectively). An increase in phospho-Akt (Thr 308) was only observed in T84 cells $(\mathrm{P}<0.001)$. Analysis of phospho-mTOR levels in Andro treated cells by immunofluorescence was consistent with phosphoAkt results (Figure 6C). There was a highly significant reduction in phospho-mTOR in both cell lines when treated with Andro $(P<0.001)$ but blocking ROS with NAC during Andro treatment significantly elevated phosphomTOR expression almost to control levels $(P<0.001)$.

\section{DISCUSSION}

The present study demonstrates the importance of ROS in Andro-induced anti-cancer cell activities. The ability of Andro to induce apoptosis and cell cycle arrest in multiple types of cancer is well documented [2], and we have recently established that Andro induced apoptosis
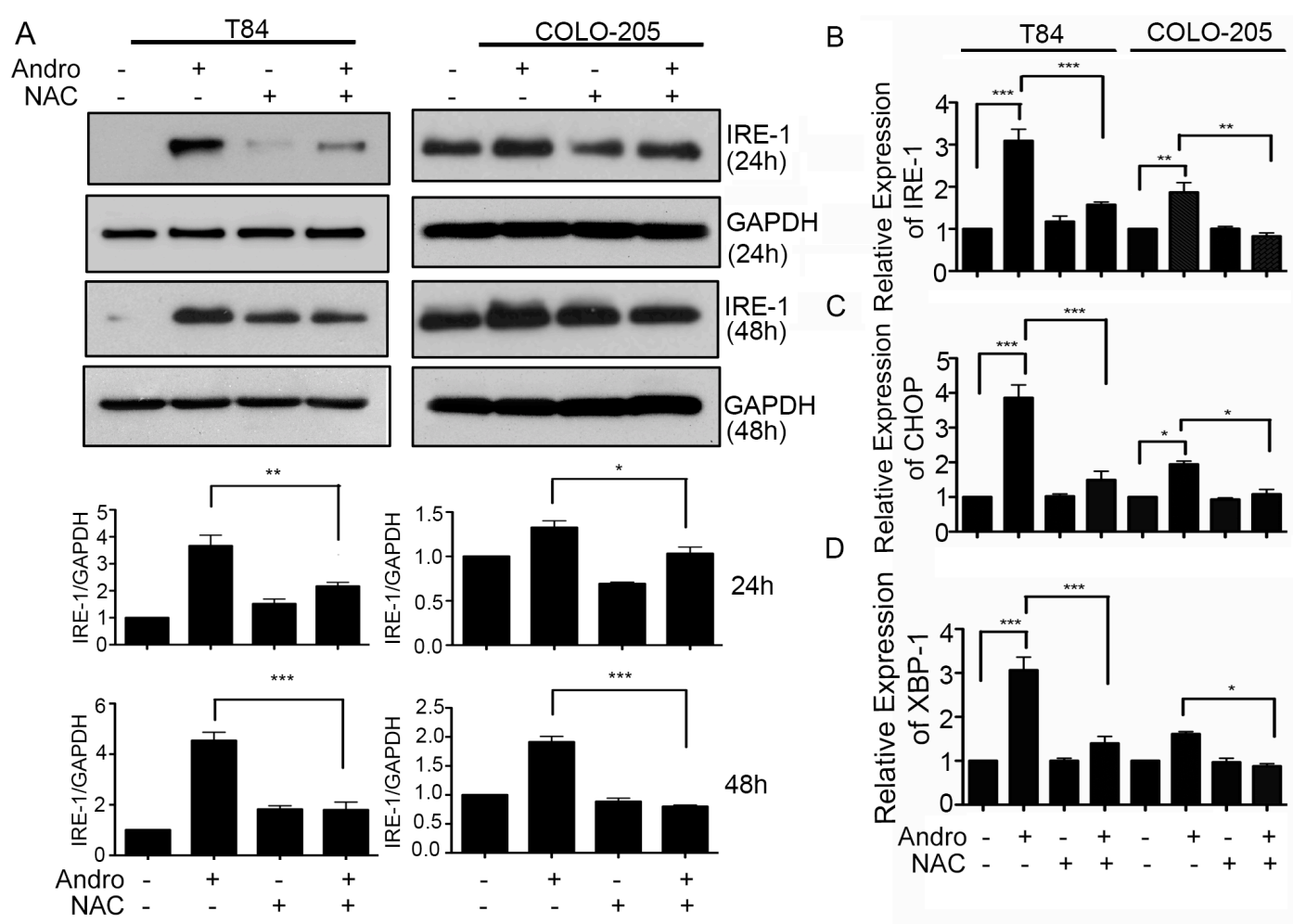

Figure 4: Andrographolide mediated ER stress and apoptosis signaling are dependent on increased ROS. (A) T84 and COLO 205 cells were pre-treated with or without NAC followed by Andro $\mathrm{IC}_{50}(45 \mu \mathrm{M})$ for $24 \mathrm{~h}$ or $48 \mathrm{~h}$. Cells were lysed and protein expression for IRE-1 and GAPDH were evaluated by immunoblot. Densitometry analysis was performed and normalized with GAPDH. Cells were also evaluated for mRNA expression by qRT-PCR for (B) IRE-1 and pro-apoptosis downstream signaling proteins, (C) CHOP, and (D) XBP-1. Results shown are from three independent experiments $(* P<0.05, * * P<0.01, * * * P<0.001)$. 
is dependent upon the induction of ER stress leading to signaling through the UPR IRE-1 protein [18]. There are multiple molecular events that can result in ER stress including oxidative stress. Our data show that treatment of colon cancer cells with Andro induces ROS production in addition to LPO, TRX, and GPX antioxidant response genes. Pretreatment of cells with the ROS scavenger NAC effectively inhibited Andro induced IRE-1expression, proapoptotic signaling, caspase 3 activity and cell death.

The apoptosis events we observed were in accordance with the early induction of ROS. We observed elevated ROS at 24 hours and although ROS levels were not determined at earlier time points, TRX and GPX antioxidant response genes were significantly elevated between four and eight hours. This is consistent with the elevated caspase 3, CHOP, and XBP-1 expression and decreased Cyclin B1 and D1 expression we documented at 24 hours as well as the beginning of nuclear fragmentation. Fragmentation increased by 48 hours at which time we also observed increased nucleosome accumulation. ROS and anti-oxidant response genes also continued to be upregulated. Several laboratories have described an Andro induced increase in ROS that is important for cell cytotoxicity in treated cancer cells [23-28]. Additionally, other plant derived molecules with anti-cancer cell activity have been shown to induce ROS that is important for apoptosis signaling $[21,29]$. Our data demonstrate that ROS production occurs upstream of ER stress and that the UPR is a crucial intermediate event in the ROS mediated anticancer cell activities of Andro.

It is noteworthy that ROS is important for two aspects of Andro mediated cell cytotoxicity, apoptosis and
A
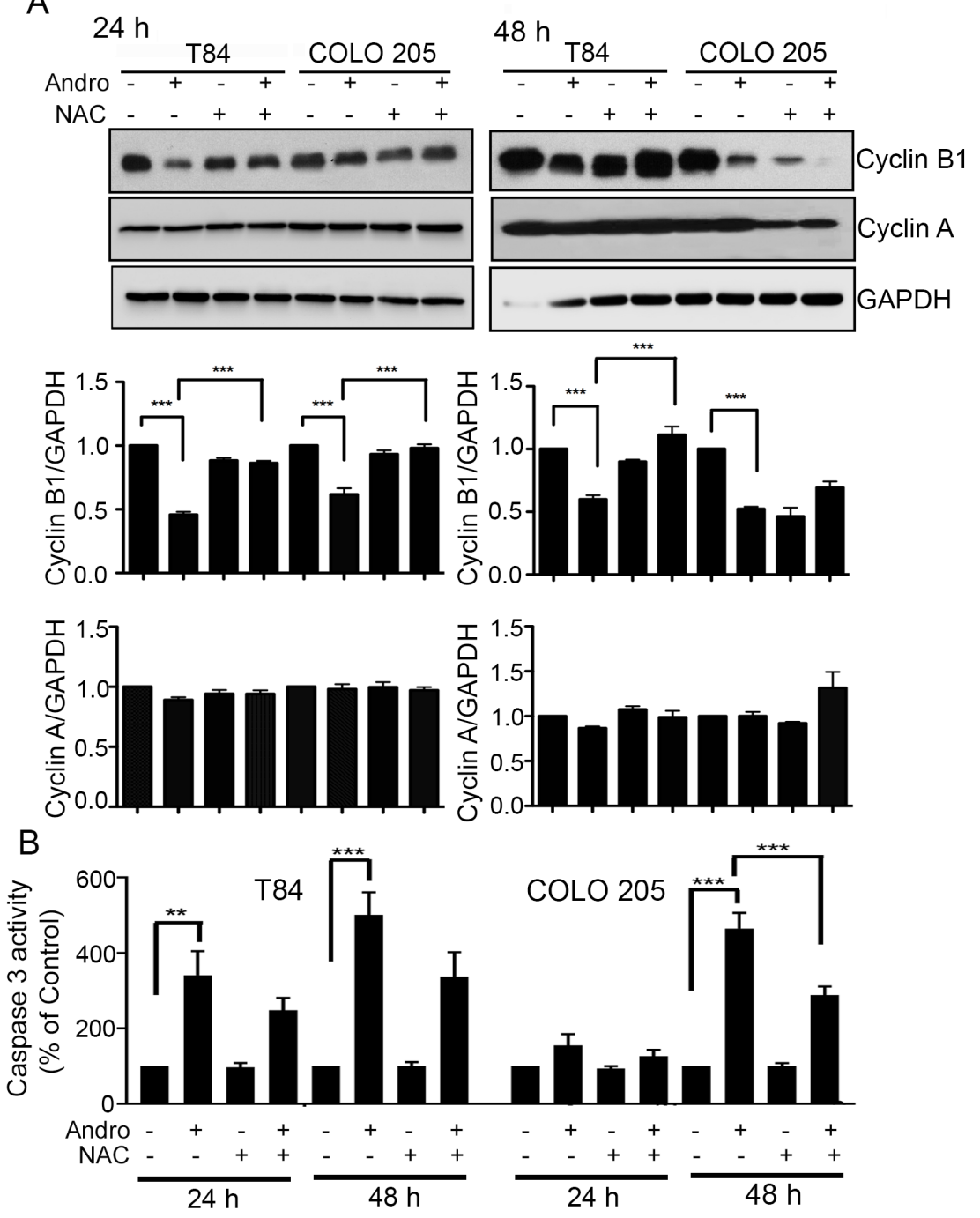

Figure 5: Andrographolide induced cell cycle arrest and apoptosis in colon cancer cells is ROS dependent. (A) T84 and COLO 205 cells were pretreated with or without NAC followed by Andro $\mathrm{IC}_{50}(45 \mu \mathrm{M})$ for $24 \mathrm{~h}$ and $48 \mathrm{~h}$. Cell lysates were analyzed by immunoblot and quantified by densitometry for expression of Cyclin B1 and cyclin A. Expression is normalized against GAPDH expression. (B) T84 and COLO 205 cells were pretreated with or without NAC followed by Andro $\mathrm{IC}_{50}$ for $24 \mathrm{~h}$ and $48 \mathrm{~h}$. Cells were then lysed and incubated with caspase 3 substrate and activity was determined by colorimetric assay at $\operatorname{OD}_{405 \mathrm{~nm}} .(* * P<0.01, * * * P<0.001)$. 
cell cycle arrest. While the pathways that lead to apoptosis can be established, the pathway leading to cell cycle arrest is less clear. The present study is consistent with several others in demonstrating down regulation of the Akt/mTOR survival pathway [15, 30-32]. Blocking this pathway can contribute to cell cycle arrest. However, the source of this down regulation has not been established. Activation of ERK has been demonstrated in some models to block Akt activation [33], but most Andro related studies report suppression or a lack of ERK activation [34-37]. There are notable exceptions where Andro associated cancer cell death was due in part to increased phopsho-ERK [26]. Alternatively, Andro may be inhibiting an event upstream of Akt activation.

In addition to cell cycle control, Akt/mTOR signaling also regulates autophagy, a process for sequestering cytosolic components and organelles for degradation by lysosomes. [38] Autophagy can also be play a role cell death, and suppression of mTOR signaling can lead to cancer cell death $[39,40]$. Similar to some other natural compounds, Andro has been demonstrated to induce autophagic cell death in several different types of cancer $[30,31,41]$. Our data demonstrating the suppression of Akt and mTOR activation in colon cancer cells are consistent with suppression of the cell survival pathway and may support autophagic cell death. The present study examined the role of ROS in the context of the recently described ER-stress/apoptosis pathway [18]. The reduced ER stress, apoptosis, and cell cycle arrest markers we observed when ROS were scavenged does not preclude the involvement of autophagy in andro-induced cell death, or the contribution of ROS towards promoting the autophagic cell death. However, unlike other cancer cell systems of andro-induced autophagic cell death in which apoptosis cell death was demonstrated to play no role, our previous studies and those of others, along with the present study demonstrate a significant role of apoptosis in andro-mediated cell death of colon cancer cells. The importance of autophagy is this system will be the subject of future investigation.

It will also be important in defining the mechanism of Andro induced cell death, to determine the source of the ROS that drives the ER stress response. While there are anti-inflammation models in which Andro is shown to suppress ROS generation by NADPH oxidase there are other models in which induced ROS levels are crucial for the apoptosis and cell cycle arrest achieved with Andro in cancer cell killing [24-28, 42]. It is likely that the ability of Andro to induce ROS is cell type dependent as Andro seems to have distinct mechanisms of action when used to treat cancer and noncancerous cells. These studies also indicate that Andro has molecular activity
A

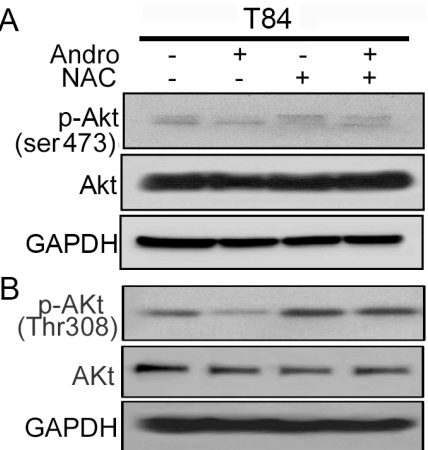

C

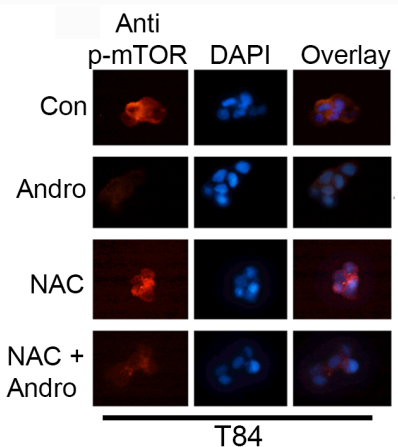

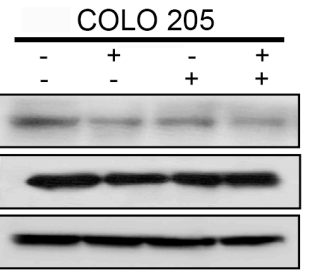

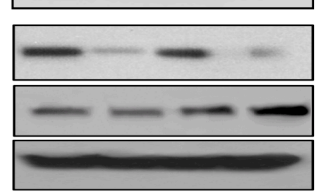

Anti

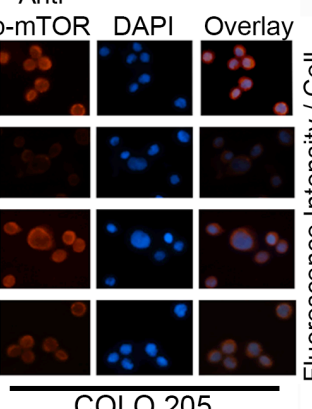

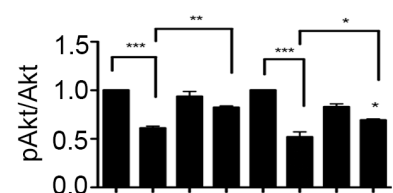
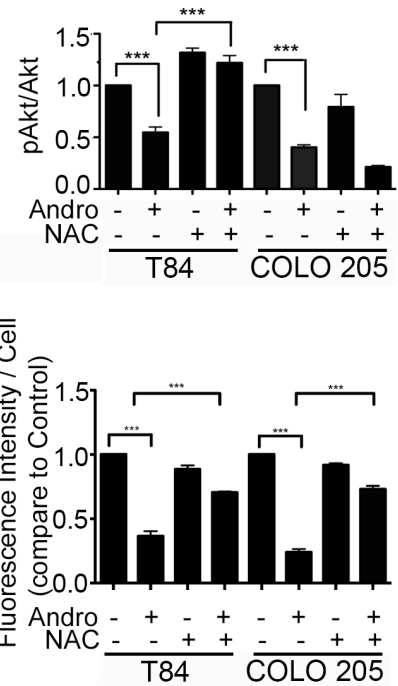

Figure 6: Andrographolide induced oxidative stress inhibits the Akt/mTOR cell survival signaling pathway. (A-B) Cell lysates from T84 and COLO 205 cells pretreated with or without NAC and then treated with Andro $\mathrm{IC}_{50}(45 \mu \mathrm{M})$ for $48 \mathrm{~h}$ were analyzed by immunoblot for expression of phospho-Akt (ser473 and Thr 308), total Akt and GAPDH and quantified by densitometry. (C) T84 and COLO 205 cells were grown on coverslips and pretreated with or without NAC, followed by Andro $\mathrm{IC}_{50}$ for $48 \mathrm{~h}$ and then phosphomTOR expression was evaluated by immunofluorescent staining. Nuclei were stained using DAPI and cells were examined by fluorescence microscopy. Fluorescence intensity was determined and compared with untreated T84 and COLO 205 cells. 
upstream of ER stress since, regardless of the source of ROS, Andro must induce molecular events that favor ROS production. It is noteworthy that a previous study documenting the importance of Andro mediated ROS in cell cytotoxicity reported the phosphorylation of the $\mathrm{p} 47$ NADPH oxidase subunit as an important event [43]. The authors hypothesized a model in which Andro activates sphingomyelinase in the cell membrane to generate ceramide which then activates membrane NADPH oxidase via phosphorylation of the p47 subunit.

The initial events of Andro molecular activity when promoting cell death in cancer cells remain ill defined despite extensive research on the effector mechanisms leading to cytotoxicity. Defining a crucial role for increased ROS production in generating ER stress leading to apoptosis, or in regulating the pathways that control cell cycle progression provides insight into initial activity of Andro that may ultimately help identify targets for improved anti-cancer therapy. While it is possible that Andro activity occurs through multiple early pathways or receptors, elucidation of the molecular events that occur when Andro first contacts a cancer cell leading to ROS production will help clarify an important aspect of Andro activity.

\section{MATERIALS AND METHODS}

\section{Cell culture and andrographolide treatment}

The T84 (ATCC ${ }^{\circledR}$ CCL-248 ${ }^{\mathrm{TM}}$ ) and COLO 205 $\left(\mathrm{ATCC}^{\circledR} \mathrm{CCL}^{-222^{\mathrm{TM}}}\right.$ ) human colon cancer cell lines, normal colon epithelial cells FHC (ATCC ${ }^{\circledR}$ CRL-1831) ${ }^{\mathrm{TM}}$ and the CCD-18Co human fibroblast cell line (ATCC ${ }^{\circledR}$ CRL-1459 ${ }^{\mathrm{TM}}$ ), were purchased from the American Type Culture Collection (Manassas, VA) and grown in the recommended complete tissue culture media. Cells were grown until approximately $75 \%$ confluent and then the media was replaced with fresh serum-free media containing $1 / 3$ the concentration of antibiotic-antifungal solution (100X stock solution used at $0.33 \mathrm{X}$ ) for $3 \mathrm{~h}$. The media was then replaced with media containing $2 \% \mathrm{FBS}$ with Andro (Sigma Aldrich, St. Louis, MO) at $\mathrm{IC}_{50}$ for 24 and $48 \mathrm{~h}$. Stock Andro was prepared in DMSO and control wells received DMSO at a final concentration of $0.01 \%$. Tunicamycin $(2 \mu \mathrm{g} / \mathrm{ml})$ was used as a positive control for ER stress induction. The inhibitor 4-PBA (100 mM) was used to block ER stress and NAC (20 mM) was used to block ROS. When these inhibitors were used, they were added $1 \mathrm{~h}$ before Andro administration.

\section{Cell viability assays}

Cell viability was assessed using the MTT assay (Sigma Aldrich) as previously described [18]. Briefly, cells were treated with the indicated concentrations of Andro in 96-well plates for indicated time points followed by the addition of MTT solution to the cells $(2.5 \mathrm{mg} / \mathrm{ml}$ final concentration). Plates were incubated at $37^{\circ} \mathrm{C}$ for $4 \mathrm{~h}$ and followed by the addition of dimethyl sulfoxide (DMSO). Optical densities $\left(\mathrm{OD}_{570 \mathrm{~nm}}\right)$ were determined on a microplate reader (Model 550, Bio-Rad, USA). Cell viability was also determined for some experiments by fluorescein diacetate (FDA; F7378, Sigma) /propidium iodide (PI; P4170, Sigma) staining according to manufacturer's instructions (Ibidi, Madison, WI). Images were taken using a fluorescence microscope.

\section{Clonogenic assay}

Cells seeded at $5 \times 10^{5} /$ well in 6 well plates were grown for $24 \mathrm{~h}$, washed, and then treated with Andro for 24 and $48 \mathrm{~h}$. Cells were then trypsinized and seeded at $5 \times 10^{3}$ cells/well in 6 well plates for 14 days. Cells were fixed with $10 \%$ buffered formalin for $15 \mathrm{~min}$, and then stained with $2 \mathrm{ml} 0.01 \%$ (w/v) crystal violet for $30 \mathrm{~min}$. Wells were washed with $\mathrm{dH}_{2} \mathrm{O}$, air dried, and colonies were counted manually by examination with an inverted microscope.

\section{DAPI staining}

Cells were evaluated by staining with DAPI (Vector Laboratories; Burlingame, CA) as previously described [44].

\section{DCFDA cellular ROS detection assay}

A DCFDA cellular ROS detection assay (\#ab113851, Abcam PLC, Cambridge, MA) was used to measure hydroxyl, peroxyl and other ROS activity within the cell. T75 flasks were seeded with $4 \times 10^{6}$ cells and grown until $75 \%$ confluent. Cells were then treated with Andro in the presence or absence of NAC as described above for $24 \mathrm{~h}$ and then washed with serum-free media. Cells were then incubated with DCFDA $(20 \mu \mathrm{M})$ at $37^{\circ} \mathrm{C}$ for $30 \mathrm{~min}$ in the dark prior to washing and manufacturer's buffer in preparation for analysis. Fluorescence intensity was determined by fluorescence spectroscopy with maximum excitation and emission spectra of 495 and 529 $\mathrm{nm}$, respectively.

\section{Measurement of mitochondrial membrane potential}

Mitochondrial transmembrane potential $\left(\Delta \Psi_{\mathrm{m}}\right)$ in T84 and COLO 205 cells was measured using a tetramethylrhodamine ethyl ester (TMRE) mitochondrial membrane potential assay kit (\#ab113852, Abcam) following the manufacturer's instructions. Briefly, $2 \times 10^{4}$ T84 and COLO 205 cells/well in a 96-well plate were challenged with the indicated dose of Andro for 24 and 48 hrs, then incubated with $500 \mathrm{nM}$ TMRE for 20 mins. The media was then gently removed and replaced with $100 \mu \mathrm{l}$ 
of PBS $/ 0.2 \%$ BSA. This step was repeated twice to remove excess dye. The fluorescence signal was quantified with Ex/ $\mathrm{Em}=549 / 575 \mathrm{~nm}$.

\section{SDS-PAGE and western blot}

SDS-PAGE and western blotting were performed as previously described $[18,45]$. The protein content for samples was determined using the BCA Protein Assay Kit (Pierce). Samples containing approximately 20-40 $\mu \mathrm{g}$ of protein were boiled in LDS sample buffer, separated on SDS-polyacrylamide gels, electrophoretically transferred to Immunoblot-P transfer membranes (IPV H00010, EMD Millipore corporation), and probed with the following primary antibodies; IRE-1 (\#3294S), p-AKt (Ser 473) (\#9271), AKt (\#9272), pAKt (Thr 308) (\#9275S), GPX (\#3206S) from Cell Signaling Technology (Danvers, MA), Cyclin B1 (GNS1, \#sc-245), cyclin D1 (\#sc-8396), cyclin A (\#H-432), PRx (A-6) P (Sc-137150P) from Santa Cruz Biotechnology (Dallas, TX), and GAPDH (\#G8795) from Sigma Aldrich. The proteins were visualized using horseradish-peroxidase-conjugated secondary antibodies (\#7074, Cell Signaling Technology) followed by Supersignal West Pico Chemiluminescent Substrate (\#34087, Thermo Scientific). Results were quantified by densitometry of digitized images using ImageJ software (NIH, Bethesda, MD, ver.1.43) and expressed as a ratio to a GAPDH loading control.

\section{Quantitative real-time polymerase chain reaction (qRT-PCR)}

Gene expression was evaluated as previously described [46]. Total RNA was purified using the EasyPure ${ }^{\circledR}$ RNA kit from Annagen Biotech (Baltimore, MD) or the High Pure RNA Isolation Kit from Roche Life Science (Indianapolis, IN) The cDNA prepared with TransScript ${ }^{\mathbb{B}}$ First Strand cDNA Synthesis Supermix (Annagen Biotech) or First Strand cDNA Synthesis Kit for RT-PCR (Roche Life Science). PCR amplification was performed with an Eppendorf Realplex Instrument (Eppendorf AG, Hamburg, Germany) with SYBR Green supermix (Fermentas, Glen Burnie, MD), $0.8 \mu \mathrm{M}$ of each primer, and $1 \mu \mathrm{l}$ cDNA. Primer sequences are listed in Supplementary Table 1. Relative gene expression changes were calculated using the $2^{-\Delta \Delta C \mathrm{~T}}$ method, and expression normalization was accomplished using housekeeping gene glyceraldehydes 3-phosphate dehydrogenase (GAPDH).

\section{Measurement of Caspase -3 activity}

Caspase-3 activity was determined by colorimetric assay according to manufacturer's instructions (\#APT165, EMD Millipore, Billerica, MA). Cells $\left(1 \times 10^{6}\right)$ were treated with Andro and NAC at the desired concentrations were incubated for $24 \mathrm{~h}$ and $48 \mathrm{~h}$. Cells were then harvested from the plates and washed by centrifuged at $1500 \mathrm{rpm}$ for $10 \mathrm{~min}$. Cells were resuspended in $100 \mu \mathrm{l}$ of chilled $1 \mathrm{X}$ cell lysis buffer and incubated on ice for 10 minutes. The cytosolic extract was recovered after centrifugation for 5 mins at $10,000 \mathrm{~g}$. Samples were mixed with assay buffer and caspase 3 substrate and then incubated for $2 \mathrm{~h}$ at $37^{\circ} \mathrm{C}$. Absorbance $\left(\mathrm{OD}_{405 \mathrm{~nm}}\right)$ was determined using a ThermoMax plate reader (Molecular Devices, Sunnyvale, CA)

\section{Fluorescence microscopy and image acquisition}

Immunofluorescence was performed as previously described [47]. Briefly, cells were grown on glass cover slips for $24 \mathrm{~h}$ and treated with or without Andro and NAC as indicated. Cells were then fixed in ice-cold methanol for $5 \mathrm{~min}$, permeabilized with $0.2 \%$ Triton $\mathrm{X}-100$ for $10 \mathrm{~min}$, blocked with $6 \%$ BSA for $30 \mathrm{~min}$ and then incubated with a 1:50 dilution of phospho-mTOR (Ser 2481) (\#2974, from Cell Signaling Technology), or TRX (\#sc-271281 from Santa Cruz), overnight at $4^{\circ} \mathrm{C}$. Cells were then incubated with Alexa Flour 594 labeled goat anti-rabbit IgG (\#A11037); or Alexa Flour 488 (\#: A-11001) from Thermo Fisher Scientific at 1:200 and DAPI for $1 \mathrm{~h}$ [18]. Sample images were taken at $200 \times$ magnification using an inverted fluorescence microscope (Olympus IX-7, Pennsylvania, USA Fluorescence intensity was quantified using ImageJ software version 1.39 (NIH). RGB composite images were created using Axion Vision rel, 4.6 and analyzed. Cells from five different fields were used for statistical analysis as previously described.

\section{Statistical analysis}

Data are presented as mean \pm standard Error (S.E). Statistical analysis was carried out with Graph Pad Prism for Macintosh 5.0c (Graph Pad Software Inc., San Diego, CA). The mean S.E. was calculated by one-way analysis of variance (ANOVA). Significance between groups was further analyzed using the post hoc Tukey's test and Bonferroni test. $P$ values were considered significant is less than 0.05 and are indicated as throughout using asterisks $* P<0.05,{ }^{* *} P<0.01, * * * P<0.001$.

\section{ACKNOWLEDGMENTS AND FUNDING}

We wish to thank the Department of Pediatrics at the University of Maryland School of Medicine for supporting this research.

\section{CONFLICTS OF INTEREST}

None of the authors participating in this study have any conflicts of interest in this work or publication. 


\section{REFERENCES}

1. World Health Organization. Herba andrographolis. In: World Health Orgaonization, ed. WHO Monographs on selected medicinal plants. (Geneva: World Health Organization). 2002.

2. Lim JC, Chan TK, Ng DS, Sagineedu SR, Stanslas J, Wong WS. Andrographolide and its analogues: versatile bioactive molecules for combating inflammation and cancer. Clin Exp Pharmacol Physiol. 2012; 39:300-310.

3. Satyanarayana C, Deevi DS, Rajagopalan R, Srinivas N, Rajagopal S. DRF 3188 a novel semi-synthetic analog of andrographolide: cellular response to MCF 7 breast cancer cells. BMC Cancer. 2004; 4:26.

4. Sukardiman H, Widyawaruyanti A, Sismindari, Zaini NC. Apoptosis inducing effect of andrographolide on TD-47 human breast cancer cell line. African journal of traditional, complementary, and alternative medicines. 2007; 4:345351 .

5. Lee YC, Lin HH, Hsu CH, Wang CJ, Chiang TA, Chen JH. Inhibitory effects of andrographolide on migration and invasion in human non-small cell lung cancer A549 cells via down-regulation of PI3K/Akt signaling pathway. Eur J Pharmacol. 2010; 632:23-32.

6. Lin HH, Tsai CW, Chou FP, Wang CJ, Hsuan SW, Wang CK, Chen JH. Andrographolide down-regulates hypoxia-inducible factor-1alpha in human non-small cell lung cancer A549 cells. Toxicol Appl Pharmacol. 2011; 250:336-345.

7. Manikam SD, Stanslas J. Andrographolide inhibits growth of acute promyelocytic leukaemia cells by inducing retinoic acid receptor-independent cell differentiation and apoptosis. J Pharm Pharmacol. 2009; 61:69-78.

8. Jada SR, Hamzah AS, Lajis NH, Saad MS, Stevens MF, Stanslas J. Semisynthesis and cytotoxic activities of andrographolide analogues. J Enzyme Inhib Med Chem. 2006; 21:145-155.

9. Kumar RA, Sridevi K, Kumar NV, Nanduri S, Rajagopal S. Anticancer and immunostimulatory compounds from Andrographis paniculata. J Ethnopharmacol. 2004; 92:291-295.

10. Ji L, Liu T, Liu J, Chen Y, Wang Z. Andrographolide inhibits human hepatoma-derived Hep3B cell growth through the activation of c-Jun N-terminal kinase. Planta Medica. 2007; 73:1397-1401.

11. Li J, Cheung HY, Zhang Z, Chan GK, Fong WF. Andrographolide induces cell cycle arrest at G2/M phase and cell death in HepG2 cells via alteration of reactive oxygen species. Eur J Pharmacol. 2007; 568:31-44.

12. Kim TG, Hwi KK, Hung CS. Morphological and biochemical changes of andrographolide-induced cell death in human prostatic adenocarcinoma PC-3 cells. In vivo. 2005; 19:551-557.

13. Zhao F, He EQ, Wang L, Liu K. Anti-tumor activities of andrographolide, a diterpene from Andrographis paniculata, by inducing apoptosis and inhibiting VEGF level. J Asian Nat Prod Res. 2008; 10:467-473.

14. Kumar S, Patil HS, Sharma P, Kumar D, Dasari S, Puranik VG, Thulasiram HV, Kundu GC. Andrographolide inhibits osteopontin expression and breast tumor growth through down regulation of PI3 kinase/Akt signaling pathway. Curr Mol Med. 2012; 12:952-966.

15. Li J, Zhang C, Jiang H, Cheng J. Andrographolide inhibits hypoxia-inducible factor-1 through phosphatidylinositol 3-kinase/AKT pathway and suppresses breast cancer growth. Onco Targets Ther. 2015; 8:427-435.

16. Lin HH, Shi MD, Tseng HC, Chen JH. Andrographolide sensitizes the cytotoxicity of human colorectal carcinoma cells toward cisplatin via enhancing apoptosis pathways in vitro and in vivo. J Toxicol Sci. 2014; 139:108-120.

17. Wong CC, Lim SH, Sagineedu SR, Lajis NH, Stanslas J. SRJ09, a promising anticancer drug lead: Elucidation of mechanisms of antiproliferative and apoptogenic effects and assessment of in vivo antitumor efficacy. Pharmacol Res. 2016; 107:66-78.

18. Banerjee A, Ahmed H, Yang P, Czinn SJ, Blanchard TG. Endoplasmic reticulum stress and IRE-1 signaling cause apoptosis in colon cancer cells in response to andrographolide treatment. Oncotarget. 2016;7:41432-44. doi: 10.18632/oncotarget.9180.

19. Hetz C. The unfolded protein response: controlling cell fate decisions under ER stress and beyond. Nat Rev Mol Cell Biol. 2012; 13:89-102.

20. Banerjee A, Lang JY, Hung MC, Sengupta K, Banerjee SK, Baksi K, Banerjee DK. Unfolded protein response is required in nu/nu mice microvasculature for treating breast tumor with tunicamycin. J Biol Chem. 2011; 286:29127-29138.

21. Kim B, Kim HS, Jung EJ, Lee JY, B KT, Lim JM, Song YS. Curcumin induces ER stress-mediated apoptosis through selective generation of reactive oxygen species in cervical cancer cells. Mol Carcinog. 2016; 55:918-928.

22. De Marchi U, Mancon M, Battaglia V, Ceccon S, Cardellini P, Toninello A. Influence of reactive oxygen species production by monoamine oxidase activity on aluminum-induced mitochondrial permeability transition. Cell Mol Life Sci. 2004; 61:2664-2671.

23. Dey SK, Bose D, Hazra A, Naskar S, Nandy A, Munda RN, Das S, Chatterjee N, Mondal NB, Banerjee S, Saha KD. Cytotoxic activity and apoptosis-inducing potential of di-spiropyrrolidino and di-spiropyrrolizidino oxindole andrographolide derivatives. PloS One. 2013; 8:e58055.

24. Liu $\mathrm{SH}$, Lin $\mathrm{CH}$, Liang $\mathrm{FP}$, Chen $\mathrm{PF}$, Kuo CD, Alam MM, Maiti B, Hung SK, Chi CW, Sun CM, Fu SL. Andrographolide downregulates the $\mathrm{v}-\mathrm{Src}$ and Bcr-Abl oncoproteins and induces Hsp90 cleavage in the ROSdependent suppression of cancer malignancy. Biochem Pharmacol. 2014; 87:229-242.

25. Yang S, Evens AM, Prachand S, Singh AT, Bhalla S, David K, Gordon LI. Mitochondrial-mediated apoptosis in lymphoma cells by the diterpenoid lactone andrographolide, 
the active component of Andrographis paniculata. Clin Cancer Res. 2010; 16:4755-4768.

26. Yang SH, Wang SM, Syu JP, Chen Y, Wang SD, Peng YS, Kuo MF, Kung HN. Andrographolide induces apoptosis of C6 glioma cells via the ERK-p53-caspase 7-PARP pathway. BioMed Res Int. 2014; 2014:312847.

27. Yuan H, Sun B, Gao F, Lan M. Synergistic anticancer effects of andrographolide and paclitaxel against A549 NSCLC cells. Pharm Biol. 2016:1-7.

28. Zhu YY, Yu G, Zhang Y, Xu Z, Wang YQ, Yan GR, He QY. A novel andrographolide derivative AL-1 exerts its cytotoxicity on K562 cells through a ROS-dependent mechanism. Proteomics. 2013; 13:169-178.

29. Tan BJ, Chiu GN. Role of oxidative stress, endoplasmic reticulum stress and ERK activation in triptolide-induced apoptosis. Int J Oncol. 2013; 42:1605-1612.

30. Hsieh MJ, Lin CW, Chiou HL, Yang SF, Chen MK. Dehydroandrographolide, an iNOS inhibitor, extracted from Andrographis paniculata (Burm.f.) Nees, induces autophagy in human oral cancer cells. Oncotarget. 2015; 6:30831-30849. doi: 10.18632/oncotarget.5036.

31. Kumar D, Das B, Sen R, Kundu P, Manna A, Sarkar A, Chowdhury C, Chatterjee M, Das P. Andrographolide Analogue Induces Apoptosis and Autophagy Mediated Cell Death in U937 Cells by Inhibition of PI3K/Akt/mTOR Pathway. PloS One. 2015; 10:e0139657.

32. Li Y, Zhang P, Qiu F, Chen L, Miao C, Li J, Xiao W, $\mathrm{Ma}$ E. Inactivation of $\mathrm{PI} \mathrm{K} / \mathrm{Akt}$ signaling mediates proliferation inhibition and G2/M phase arrest induced by andrographolide in human glioblastoma cells. Life Sci. 2012; 90:962-967.

33. Zhuang S, Schnellmann RG. A death-promoting role for extracellular signal-regulated kinase. J Pharm Exp Ther. 2006; 319:991-997.

34. Chao CY, Lii CK, Hsu YT, Lu CY, Liu KL, Li CC, Chen HW. Induction of heme oxygenase-1 and inhibition of TPA-induced matrix metalloproteinase-9 expression by andrographolide in MCF-7 human breast cancer cells. Carcinogenesis. 2013; 34:1843-1851.

35. Chao HP, Kuo CD, Chiu JH, Fu SL. Andrographolide exhibits anti-invasive activity against colon cancer cells via inhibition of MMP2 activity. Planta Medica. 2010; 76:1827-1833.

36. Liang FP, Lin CH, Kuo CD, Chao HP, Fu SL. Suppression of $\mathrm{v}-\mathrm{Src}$ transformation by andrographolide via degradation of the $\mathrm{v}$-Src protein and attenuation of the Erk signaling pathway. J Biol Chem. 2008; 283:5023-5033.

37. Yang T, Yao S, Zhang X, Guo Y. Andrographolide inhibits growth of human T-cell acute lymphoblastic leukemia Jurkat cells by downregulation of PI3K/AKT and upregulation of p38 MAPK pathways. Drug Des Dev Ther. 2016; 10:1389-1397.

38. Klionsky DJ. Autophagy: from phenomenology to molecular understanding in less than a decade. Nat Rev Mol Cell Biol. 2007; 8:931-937.

39. Kondo Y, Kanzawa T, Sawaya R, Kondo S. The role of autophagy in cancer development and response to therapy. Nat Rev Cancer. 2005; 5:726-734.

40. Suh Y, Afaq F, Khan N, Johnson JJ, Khusro FH, Mukhtar H. Fisetin induces autophagic cell death through suppression of mTOR signaling pathway in prostate cancer cells. Carcinogenesis. 2010; 31:1424-1433.

41. Chen W, Feng L, Nie H, Zheng X. Andrographolide induces autophagic cell death in human liver cancer cells through cyclophilin D-mediated mitochondrial permeability transition pore. Carcinogenesis. 2012; 33:2190-2198.

42. Lu H, Zhang XY, Wang YQ, Zheng XL, Yin Z, Xing WM, Zhang Q. Andrographolide sodium bisulfate-induced apoptosis and autophagy in human proximal tubular endothelial cells is a ROS-mediated pathway. Environ Toxicol Pharmacol. 2014; 37:718-728.

43. Chen YY, Hsu MJ, Sheu JR, Lee LW, Hsieh CY. Andrographolide, a Novel NF- kappa B Inhibitor, Induces Vascular Smooth Muscle Cell Apoptosis via a Ceramidep47phox-ROS Signaling Cascade. Evid Based Complement Alternat Med. 2013; 2013:821813.

44. Majumdar KN, Banerjee A, Ratha J, Mandal M, Sarkar RN, Saha KD. Leishmanial lipid suppresses tumor necrosis factor alpha, interleukin-1beta, and nitric oxide production by adherent synovial fluid mononuclear cells in rheumatoid arthritis patients and induces apoptosis through the mitochondrial-mediated pathway. Arthritis Rheum. 2008; 58:696-706.

45. Nita-Lazar M, Banerjee A, Feng C, Vasta GR. Galectins regulate the inflammatory response in airway epithelial cells exposed to microbial neuraminidase by modulating the expression of SOCS1 and RIG1. Mol Immunol. 2015; 68:194-202.

46. Shiu J, Piazuelo MB, Ding H, Czinn SJ, Drakes ML, Banerjee A, Basappa N, Kobayashi KS, Fricke WF, Blanchard TG. Gastric LTi cells promote lymphoid follicle formation but are limited by IRAK-M and do not alter microbial growth. Mucosal Immunol. 2015; 8:1047-1059.

47. Nita-Lazar M, Banerjee A, Feng C, Amin MN, Frieman MB, Chen WH, Cross AS, Wang LX, Vasta GR. Desialylation of airway epithelial cells during influenza virus infection enhances pneumococcal adhesion via galectin binding. Mol Immunol. 2015; 65:1-16. 